\title{
Correlated Roughness in Polymer Films Containing Maghemite Nanoparticles
}

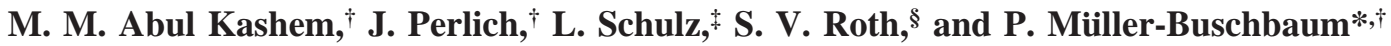 \\ Physik Department LS E13, TU München, James-Franck-Strasse 1, 85747 Garching, Germany, Physics \\ Department, Université de Fribourg, Chemin du Musée 3, 1700 Fribourg, Switzerland, and HASYLAB \\ at DESY, Notke Strasse 85, 22603 Hamburg, Germany
}

\begin{abstract}
Two different uniform thicknesses $(8$ and $68 \mathrm{~nm})$ of a nanocomposite film comprising diblock copolymer matrix and magnetic nanoparticles filler have been installed in one sample preparation step under identical condition simultaneously. Long-range interface correlations are investigated. Maghemite $\left(\mathrm{F}_{2} \mathrm{O}_{3}\right)$ nanoparticles covered with polystyrene chains are incorporated in polystyrene-block-polyisoprene to produce such film. The film is investigated with atomic force microscopy (AFM), X-ray reflectivity (XR), and scanning microfocus grazing incident small-angle X-ray scattering (GISAXS). The roughness of the substrate is replicated on the $68 \mathrm{~nm}$ thick composite film surface resulting in roughness correlation in a waveguide manner. No roughness correlation is observed in $8 \mathrm{~nm}$ film.
\end{abstract}

\section{Introduction}

Polymeric nanocomposite materials, which consist of polymer matrix and inorganic fillers, have the advantages of achieving desired mechanical, electrical, and thermal properties. ${ }^{1-5}$ The widely used fillers include carbon black, clay, silica, and carbon nanotubes. The surface topography and arrangement of fillers inside the matrix of thin films influence typical properties of nanocomposites like magnetic properties, which can be rendered to the polymer by using magnetic nanoparticles in the diblock copolymer film. ${ }^{6-15}$ The spin-coating technique is frequently used to prepare such kind of nanocomposite films of constant thickness on top of solid support. A broad range of film thicknesses (from the submonolayer regime up to several micrometers) are accessible by varying the preparation parameters such as the concentration of the polymer solution, the molecular weight of the polymer and the rotation speed. ${ }^{16}$ The film characteristics are strongly dependent on the interfacial energy of substrate and the film, film thickness and drying kinetics during spin-coating. The film can be completely wetted, partially dewetted, or fully dewetted. Preventing dewetting of thin and ultrathin film is a big issue. Barnes and Karim et al. used C60 fullerene to suppress the dewetting process in homopolymer. ${ }^{17,18}$ Krishnan and Mackay et al. reported the similar effects using dendrimer and PS nanoparticles. ${ }^{19,20}$ McGarrity et al. reported recently that the inhibition of dewetting due to added nanoparticles to the supported thin polymer films occurs because of migration of nanoparticles to the substrate. ${ }^{21}$ Therefore, the film thickness of a nanocomposite, either partially dewetted or wetted film, which is produced by spin-coating, is statistically an average value. Local deviations from this mean value are given by the roughness. This means the local thicknesses at some positions are smaller than the average film thickness. This might lead to an inefficient protective strength at such a spot of smaller thickness of the film for very special technical application such as coatings in microelectronics and

* Corresponding author. Telephone: +49 89289 12451. Fax: +49 89 28912473. E-mail: muellerb@ph.tum.de.

$\dagger$ Physik Department LS E13, TU München.

Physics Department, Université de Fribourg.

$\S$ HASYLAB at DESY. optics. With respect to application these deviations force the necessity to account for this. The installation of long-range interface correlations offers an approach to overcome this problem.

Because the solid substrate has a given topography the film should exhibit the similar topography to have a locally welldefined film thickness. This replication of topography is identical with a replication of the roughness spectrum from the solid substrate through a thin film surface. It results in a correlation among both..$^{22-28}$ One extreme case is a perfect correlation between substrate and film, which is called "conformal" roughness. This phenomenon has been observed in hard matter film. Such kind of film is called fully correlated. In the other extreme case, the roughness spectrum is not replicated on top of the film surface. It is called uncorrelated. The behavior of polymer chains is found to behave in between both limiting cases. The polymer chains cannot follow completely the roughness spectrum of the substrate and only the larger wavelengths are correlated. Therefore, typically polymers can form partially correlated films. Figure 1 shows schematically these three kinds of roughness correlations between the thin film and the substrate underneath.

Andelmann et al. ${ }^{29,30}$ investigated theoretically in the framework of linear response theory how the Fourier spectrum of an absorbed thin film is influenced by a rough solid substrate. Later V. Holý and T. Baumbach studied the interface roughness of a periodical AlAs/GaAs multilayer using non specular X-ray reflectivity and showed a good agreement with the theoretical calculation based on distorted-wave Born approximation (DWBA). ${ }^{31}$ In literature, interface correlation was also observed in multilayer of hard matter and soft polymers and in single layered films, ${ }^{22-26}$ Langmuir-Blodgett films, ${ }^{27,28}$ and smectic films. ${ }^{32,33}$ It was observed, that roughness correlation in thin polymer films decays with a long annealing time. ${ }^{23}$ Below the entanglement molecular weight no long-ranged interface correlation was observed. $^{23}$ However, at high molecular weights the chain structure tends to weaken roughness correlation as well. Thus, to obtain roughness correlation, i.e., a locally well-defined film thickness an intermediate regime of molecular weights seems optimal. To the best of our knowledge, so far there is no report 


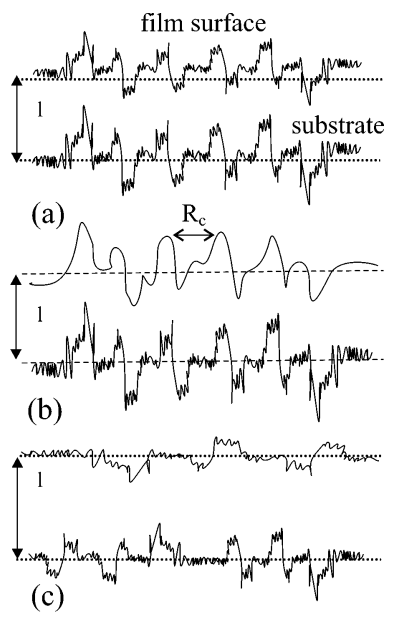

Figure 1. Schematic side view showing the replication of the roughness spectrum from a substrate to the top of the film. Key: (a) fully correlated; (b) partially correlated; (c) uncorrelated. $l$ denotes the mean film thickness, which is usually determined by standard X-ray reflectivity or ellipsometry measurements. The in-plane cutoff length $R_{\mathrm{c}}$ determines the smallest length of the roughness spectrum, which is replicated by the film, i.e., only in-plane length scales larger than $R_{\mathrm{c}}$ are transferred to the film interface. The amplitude of the roughness spectrum is highly exaggerated for clarity of the presentation.

on roughness correlation in polymer composite films containing magnetic nanoparticles.

Within the present investigation we focus on roughness correlation between a Si substrate and nanocomposite film with a diblock copolymer matrix and embedded maghemite nanoparticles. We address two different film thicknesses. These two thicknesses are installed in one film within one single preparation step simultaneously, under identical condition for a better comparison. It is important to have two different thicknesses of such film because the arrangement of particles varies with the film thicknesses and can be used for different application purpose.$^{8-11}$ Roughness correlation depends on the arrangement of the particles. The surface topography of such film is investigated by atomic force microscopy (AFM) and the average film thickness is measured by X-ray reflectivity (XR) measurement. To be able to access the film of two different thicknesses, the investigation of lateral structures was carried out by scanning microfocus grazing incident small-angle X-ray scattering (GISAXS). The grazing incidence geometry enhances the surface sensitivity and overcomes the limitations of conventional small-angle X-ray scattering studies regarding extremely small sample volumes in the thin film geometry. ${ }^{34}$

\section{Experimental Section}

Sample Preparation. The investigated sample was prepared from a symmetric diblock copolymer polystyrene-block-polyisoprene, denoted as $\mathrm{P}(\mathrm{S}-b-\mathrm{I})$, with a molecular weight $M_{\mathrm{w}}=24500$ $\mathrm{g} / \mathrm{mol}$ having a volume fraction of PS $f_{\mathrm{PS}}=N_{\mathrm{PS}} / N=0.56$. This polymer has a lamellar morphology in the bulk. Maghemite nanoparticles $\left(\mathrm{Fe}_{2} \mathrm{O}_{3}\right)$ with a mean hydrodynamic diameter of 11 $\mathrm{nm}$ (in toluene solution) and a solid core with a mean diameter of $8 \mathrm{~nm}$, with $20 \%$ size distribution (fwhm), coated with polystyrene chains were used as filler in the polymer matrix. A polymer solution (polymer concentration is $10 \mathrm{mg} / \mathrm{mL}$ in toluene) containing $25 \%$ (wt) nanoparticles as a dispersion was used for the film preparation. Two different thicknesses in the same film were installed in one single step of processing.

At first, we cleaned the substrate by using an acidic bath (160 $\mathrm{mL}$ of $96 \%$ sulfuric acid, $70 \mathrm{~mL}$ of $30 \%$ hydrogen per oxide and $110 \mathrm{~mL}$ of deionized water) at $80{ }^{\circ} \mathrm{C}$ followed by strong rinsing with deionized water immediately before coating. Next, the substrate was partially covered with another piece of clean silicon wafer piece. The polymer solution containing the nanoparticles was poured on top of the uncovered part of the substrate. The film was produced then by spin-coating ( $2000 \mathrm{rpm}$ for $30 \mathrm{~s}$ ). The produced film contains two parts of different thicknesses separated by a thickness gradient with a rim shape. The part of the substrate, which was uncovered in the spin-coating, contains the film of $68 \mathrm{~nm}$ thickness. The part of the substrate, which was covered with the second piece of $\mathrm{Si}$, contains a discontinuous film of average height of $\sim 8 \mathrm{~nm}$. Near the border between the uncovered and covered part a rim of $\sim 700 \mathrm{~nm}$ height is produced. No additional processing was applied.

Atomic Force Microscopy. The surface topography was imaged by noncontact atomic force microscopy. We used an Autoprobe CP Research AFM instrument. In noncontact mode the tip-induced damage was minimized. We used gold coated silicon cantilevers (Ultralever cantilevers), which had a resonance frequency of 60 $\mathrm{kHz}$, a tip with a high aspect ratio and an asymptotic conical shape. The radius of curvature of the tip was approximately $10 \mathrm{~nm}$ which is small as compared to the structures measured. The measurements were performed at room temperature in air. The AFM height and lateral calibration was performed several times with calibration standards to improve the accuracy of the height and lateral information. This calibration worked over the covered range of heights and surface area due to the hardware linearization of our AFM system. Only topographical information was obtained in noncontact mode of operation. No phase shift information was recorded. The measurements were carried out at different positions of the sample with different scan sizes (typically 1, 2, 4, 8, 15, 30, and $50 \mu \mathrm{m}$ ). Each scanned micrograph consists of 256 lines, scanned with $0.25 \mathrm{~Hz}$ up to $1.0 \mathrm{~Hz}$. Tapping mode gave us phase contrast among hard solid surface, hard nanoparticles and soft polymer. The background due to the scanner-tube movement was fully subtracted from the raw data.

X-ray Reflectivity. The film thickness and their internal compositions were determined by X-ray reflectivity measurement and fitting with Parratt algorithm ${ }^{35,36}$ using a model system. The measurement was carried out with the laboratory $\mathrm{X}$-ray source instrument (X-ray reflectometer/diffractometer D5000 from Siemens). The instrument consists of a sample stage, a knife edge collimator (KEC) and an automatic beam absorber. The X-rays (wavelength $\lambda=0.154 \mathrm{~nm}$ from the $\mathrm{Cu}$ target) were collimated by a slit system which was adapted in order to run the experiment with a proper beam divergence. A graphite monochromator was used in the reflected beam path, in front of the detector to filter the $\mathrm{Cu} \mathrm{K}_{b}$ lines of the source. A scintillation counter detector was used to record the reflectivity data. The automatic beam absorber was used to reduce the beam intensity to avoid saturation of the detector. The sample was placed on the sample stage and fixed by vacuum chuck. The position of the sample, beam direction, and footprint of the beam were adjusted with care so that the reflectivity data does not show any average information on the different parts (thin, ultrathin, or rim) of the film. A typical $\theta-2 \theta$ scan was performed where $2 \theta$ was varied from $0^{\circ}$ to $6^{\circ}$.

GISAXS. The roughness correlation of such kind of film, having two regions of different thicknesses, was examined with the surface sensitive X-ray scattering technique grazing incident small-angle X-ray scattering (GISAXS). The basic setup of the GISAXS scattering geometry is shown schematically in Figure 2. Scanning microfocus GISAXS measurements were carried out at the beamline BW4 of the DORIS III storage ring at HASYLAB (DESY, Hamburg). The selected wavelength was $\lambda=0.138 \mathrm{~nm}$. The beam divergence in and out of the plane of reflection was set by two entrance cross-slits. The beam was focused to the size of $(H \times V)$ $60 \times 30 \mu \mathrm{m}^{2}$ by using an assembly of refractive beryllium lenses. ${ }^{37}$ The sample was placed horizontally on a goniometer. A beam stop was used to block the direct beam in front of the detector. In addition, a second, point-like moveable beam stop was also used to block the specular peak intensities on detector. The incident angle was $\alpha_{i}=0.72^{\circ}$, which is well above the critical angles of the diblock polymer and the nanoparticles. In this geometry the beam penetrated the full film and thus the scattering data gave access to 


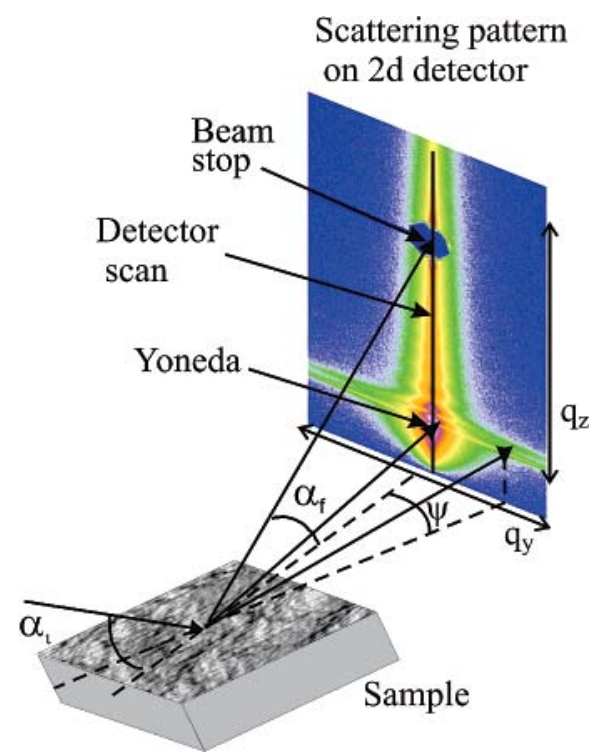

Figure 2. Experimental setup in GISAXS geometry is shown schematically: The sample surface is placed horizontally. The incident angle is denoted as $\alpha_{\mathrm{i}}$, the exit angle as $\alpha_{\mathrm{f}}$, and the out-of-plane angle as $\psi$. The two-dimensional detector resembles the $q_{y}$ dependence along the horizontal axis and the $\mathrm{q}_{z}$ dependence (neglecting small $q_{x}$ dependence) along the vertical axis. The scattering intensity distribution on the detector (low intensity as dark and high intensity as bright) is presented in a logarithmic scale. The detected scattering pattern shows the diffuse scattering with modulations of the intensity between the Yoneda peak and the specular peak (shielded by a beam-stop that protects the detector from a very high intensity of the reflected beam).

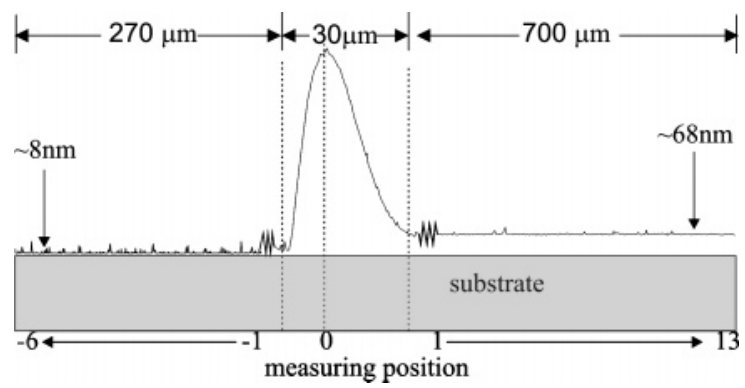

Figure 3. Scheme of the scanning GISAXS measurement with a figuratively shown thickness profile of the measured area as probed with AFM. The position of the rim is considered as 0 . The ultrathin part of the film is on the left side of the rim having the measuring position -6 to -1 . The thin part is on the right side of the rim and the measured positions are assigned as positive from 1 to 13 . The total measured length of ultrathin part is $\sim 270 \mu \mathrm{m}$, rim is $\sim 30 \mu \mathrm{m}$ and thin part is $\sim 700 \mu \mathrm{m}$. The rim height is $\sim 700 \mathrm{~nm}$, the thickness of ultrathin part is $\sim 8 \mathrm{~nm}$, and the thin part is $68 \mathrm{~nm}$.

the information from inside the film and not only from the polymer films surface, which is essential to probe correlations between the substrate surface and the polymer film surface. The scattered intensity was recorded on a two-dimensional (2D) detector (MARCCD; $2048 \times 2048$ pixel) positioned at $D_{\mathrm{SD}}=1.97 \mathrm{~m}$ behind the sample.

Both parts of the film, the thin and the ultrathin one, were investigated with scanning the sample with respect to the X-ray beam in GISAXS geometry. Starting from the ultrathin part the measurement continued to the thin part of the film through the thickness gradient rim. In total a region of $1 \mathrm{~mm}$ length was scanned. It was measured in 20 steps with $50 \mu \mathrm{m}$ scan-step size. The total measurement scheme is explained schematically in Figure 3. In Figure 3, the distances are given as relative distances measured from the rim position. Therefore, the rim is positioned at 0 . The scanning started at position -6 and ended at position 13. Approximately $270 \mu \mathrm{m}$ of the ultrathin part, $30 \mu \mathrm{m}$ of the rim, and $700 \mu \mathrm{m}$ of the thin part were probed in this investigation.
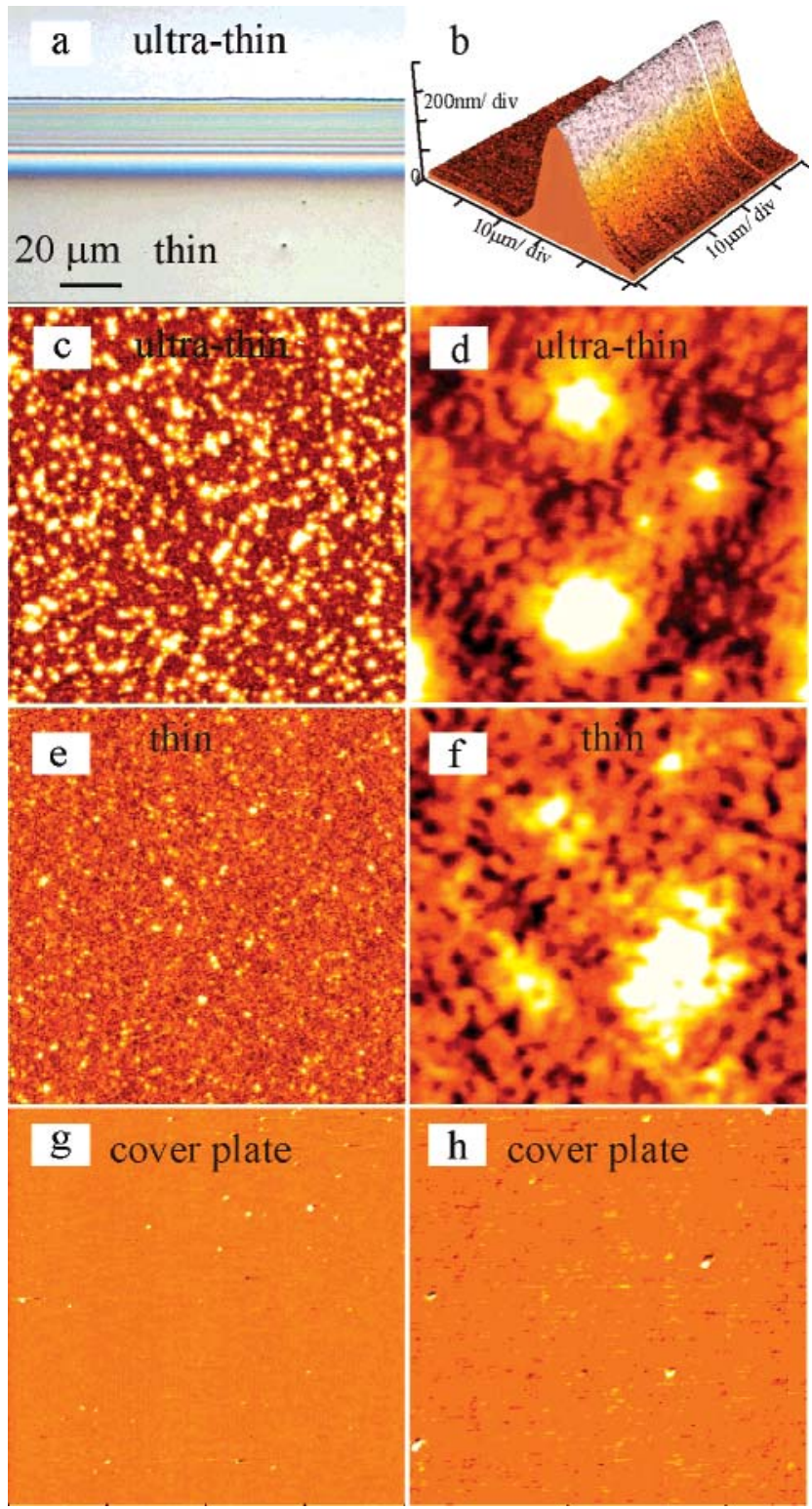

Figure 4. Surface topography at different length scale showing the thin part, the rim and the ultrathin part of the film and the cover plate. (a) Optical microscopic top view $(50 \times$ zoom $)$ of the film showing the ultrathin part and thin part of the film separated by thickness gradient rim (in the middle of the image color changes with thickness in gradient). Spot containing big dust particles are selected to show that the focus of the microscope was on the film. (b) $3 \mathrm{~d}$ view of the thickness gradient $\operatorname{rim}(50 \mu \mathrm{m} \times 50 \mu \mathrm{m})$ having ultrathin part on the left side and thin part on the right side obtained by AFM. $(\mathrm{c}-\mathrm{h})$ AFM height images of ultrathin, thin part of the film and cover plate of different scan sizes $(8 \mu \mathrm{m} \times 8 \mu \mathrm{m}$ for $\mathrm{c}$, e, and $\mathrm{g} ; 1 \mu \mathrm{m} \times 1 \mu \mathrm{m}$ for $\mathrm{d}$, $\mathrm{f}$, and h) showing the local surface structures at different length scales. The gray scales cover a range from 0 to $15 \mathrm{~nm}$ for $\mathrm{c}$ and e, from 0 to $9 \mathrm{~nm}$ for $\mathrm{d}$, from 2 to $7 \mathrm{~nm}$ for $\mathrm{f}$, from 0 to $3 \mathrm{~nm}$ for $\mathrm{g}$ and from 0 to $1 \mathrm{~nm}$ for $h$. The bright structures in images of the ultrathin part (c and d) are nanoparticle clusters and in image $\mathrm{f}$ are mostly polymeric structures.

\section{Results and Discussion}

1. Surface Topography. The surface topography of the sample was investigated by optical microscopy in reflection mode and by atomic force microscopy in noncontact mode. Optical microscopy yields the overall top view of the film describing the large surface structures. Figure 4 shows the surface topography at different length scales. The gradual changes in color in the middle of the optical image (Figure 4a) represent the thickness gradient rim which separates the ultrathin and the thin part of the film. Two different colors of these two 


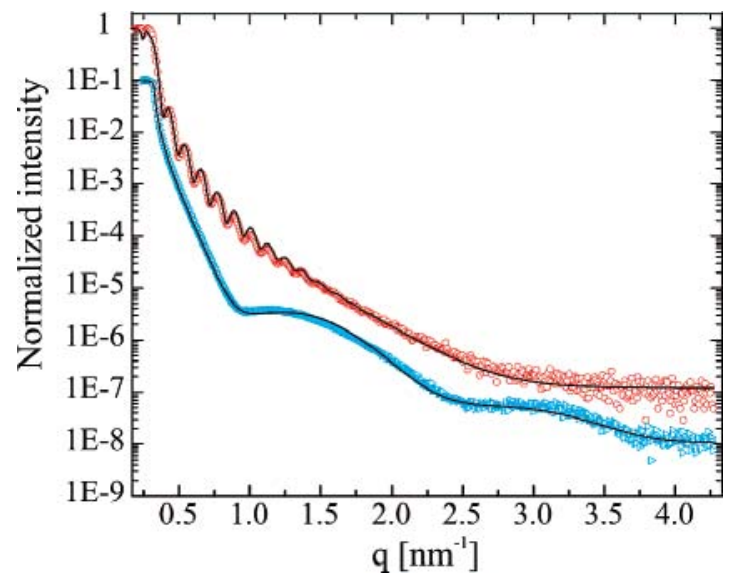

Figure 5. X-ray reflectivity data measured on the ultrathin (triangle) and thin (circle) part of the film. The solid lines are the fits used to calculate the thickness, interfacial roughness and perpendicular structures of the film. The intensities are normalized to their highest intensity. The data of ultrathin part is shifted by 1 order of magnitude downward for better visualization.

parts are distinct and represent two different homogeneous thicknesses of the film. To ensure focusing on the film surface an area with dust particles was selected for presentation. The optical image cannot give the detail information about the surface structures present on the surface, which have the dimensions of a few nanometers only. AFM in noncontact mode was used to observe the local structures on the surface. Noncontact mode was used to avoid the tip induced damage/ creation of the surface structures. As a result, no phase contrast image was obtained in noncontact mode. To distinguish the hard nanoparticles from the soft polymer a few number of measurements were also carried out in tapping mode with special care. But these images are not presented in this article. Figure $4 \mathrm{~b}$ shows the $3 \mathrm{~d}$ view of the rim part of the film obtained by AFM with $50 \mu \mathrm{m} \times 50 \mu \mathrm{m}$ scan size. The thickness of the left part is $\sim 8 \mathrm{~nm}$ and that of right part is $\sim 68 \mathrm{~nm}$. These thicknesses were measured by making a scratch on the film and measuring the height difference between the scratch and the film. From the scratch techniques, we obtain only a preliminary estimation of the thickness. The left part of the rim is denoted with ultrathin and the right part with thin.

(a) Ultrathin Part. The ultrathin part of the film is not a continuous regular film. The dark areas on the AFM images (Figure 4, parts c and d) are picturing the solid substrate and the brighter white structures represent nanoparticles and their clusters. All other structures visible in Figure 4, parts c and d, are formed by the diblock copolymer. Thus, the polymeric structures are worm-like and support the nanoparticles and nanoparticle clusters (see Figure 4, parts c and d). The structure is formed during the spin-coating process in the narrow opening between the two $\mathrm{Si}$ wafers, one acting as the substrate and the other acting as the shield. Because no further annealing was applied, the observed structures are non-equilibrium structures, frozen-in during the solvent evaporation during the spin-coating process. The opening is installing a confinement. It is so small that it is filled by only a very small amount of copolymernanoparticle solution. Thus, not enough material is present to form a uniform film and to prevent the onset of dewetting. Although nanoparticles are well-known to prevent dewetting, ${ }^{38-46}$ in the ultrathin film case nanoparticles retard dewetting. ${ }^{9}$ Recently, a similar kind of structure was reported in the case of an ultrathin film using similar materials. ${ }^{9}$ In addition, the structures show no sign of fracture introduced features due to the lift-off of the cover plate.
Table 1. Parameters Used to Fit the X-ray Reflectivity Data Shown in Figure 6

\begin{tabular}{lcccccc}
\hline & & & & \multicolumn{3}{c}{ composition (vol \%) } \\
\cline { 5 - 7 } $\begin{array}{c}\text { part of } \\
\text { the film }\end{array}$ & $\begin{array}{c}\text { layer } \\
\text { abbrev }\end{array}$ & $\begin{array}{c}\text { thickness } \\
(\mathrm{nm})\end{array}$ & $\begin{array}{c}\text { roughness } \\
(\mathrm{nm})\end{array}$ & $P(\mathrm{~S}-b-\mathrm{I})$ & $\mathrm{Fe}_{2} \mathrm{O}_{3}$ & air \\
\hline \multirow{2}{*}{ ultrathin } & $h 1$ & $3.7 \pm 0.2$ & $1 \pm 0.1$ & 0 & 36.5 & 63.5 \\
& $h 2$ & $4.2 \pm 0.3$ & $0.3 \pm 0.02$ & 63.5 & 36.5 & 0 \\
\multirow{4}{*}{ thin } & $\mathrm{Si}$ & & 0.1 & & & \\
& $h 3$ & $51.0 \pm 1.0$ & $0.7 \pm 0.05$ & 97.3 & 2.7 & 0 \\
& $h 4$ & $17.0 \pm 2.0$ & $0.3 \pm 0.02$ & 64.3 & 35.7 & 0
\end{tabular}

(b) Thin Part. On the uncovered part of the substrate a homogeneous copolymer film with embedded nanoparticles is present. Exactly the same film forms on a simple Si substrate (without the applied shielding technique) and its thickness is controlled by the polymer concentration used for the spincoating. The thin part of the film has similar kind of surface topography regarding the structures formed by the copolymer (see Figure 4, parts e and f). However, the holes in the copolymer film surface are only due to islands because the material was insufficient to form a complete uniform layer. The holes do not have the depth of the total film, i.e., do not show the substrate underneath but polymer film. Because no microphase separated structures were found, the hole-depth does not give any characteristics length such as the bulk lamellar spacing. The copolymer film is a disordered state. Only a very few nanoparticles clusters were found on top of the surface by AFM. Thus, most particles are embedded in the film. It was enough polymer material to cover the nanoparticles and their formed clusters. Before starting the spin-coating, sedimentation starts the migration of the nanoparticles toward the substrate surface. During spin-coating the migration is stopped by centrifugal force and the increase of viscosity of the polymer solution because of solvent evaporation. This leads to a nonuniform distribution of particles inside the film. Near the substrate the concentration of the particles is expected to be higher than that of particles near the air-film interface. In this case the presence of nanoparticles and enough polymer materials retarded dewetting. Therefore, a continuous film was produced. The structures on the surface might be produced during the evaporation of the solvent.

(c) The Cover Plate. The surface topography of the ultrathin part of the film might be affected by sticking of polymer on the cover plate. Therefore, the topography of the cover plate has been investigated after removal. No special features that can influence the surface structure of the ultrathin part are found supporting that no fracture occurred while lifting-off the cover plate. The rms roughness of the cover plate is determined by the AFM image analysis software IP1.3 to be $0.1 \mathrm{~nm}$. Therefore, the surface is quite smooth, and no polymer is sticking on it. The possible explanation might be that the evaporation of the solvent, taking place in between both plates, prevents sticking of the materials on top plate but forming the structures on the bottom substrate only.

The statistical height information on the surface in the ultrathin and the thin film part can be explained by the rms roughness. ${ }^{47}$ The rms roughnesses of both parts are calculated from $8 \mu \mathrm{m} \times 8 \mu \mathrm{m}$ size images with the AFM image analysis software IP1.3. The values of rms roughnesses are 1.04 and $1.06 \mathrm{~nm}$ for the thin and ultrathin part of the film respectively excluding the big clusters present on top of the surface.

2. Film Depth Profile. Film thickness plays a very important role in the case of interface correlation. With increasing film thickness the correlation decreases and only the long wave length 


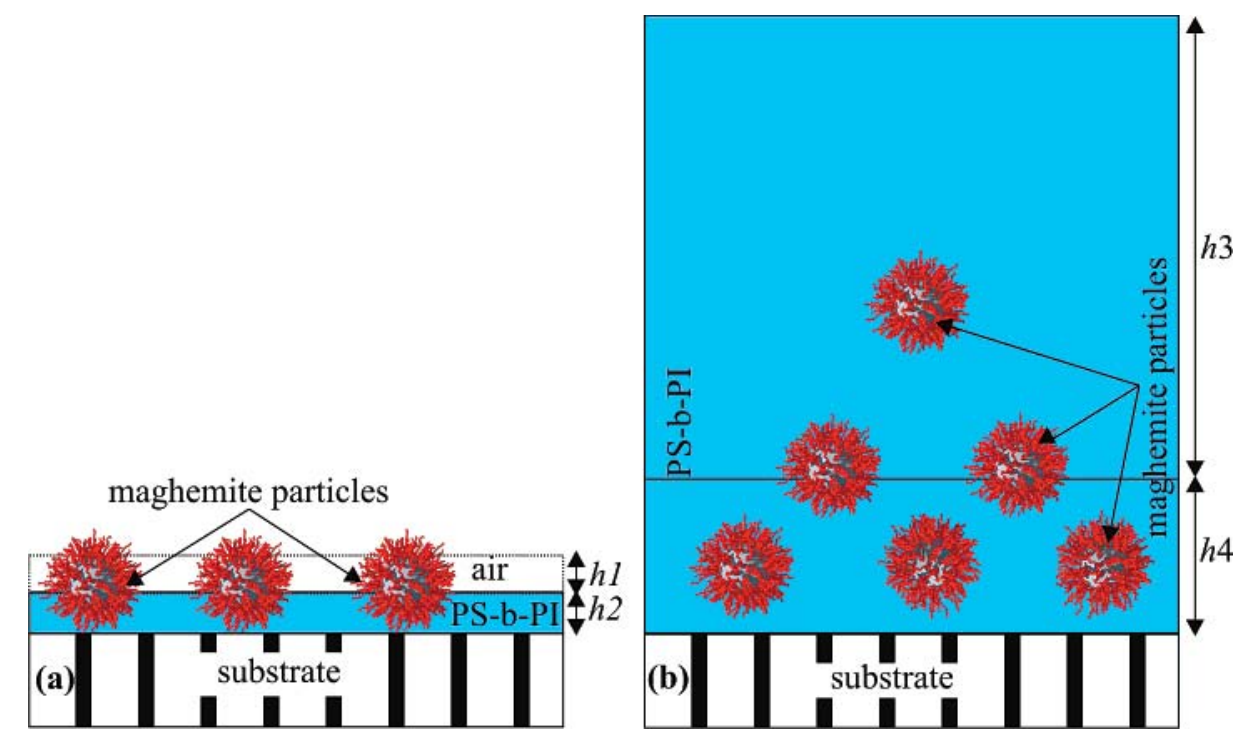

Figure 6. Rough sketch of the model used to fit the X-ray reflectivity data neglecting the roughnesses, particle clusters, and holes and island on top of surface. (a) ultrathin film and (b) thin film. h1, h2, h3, and h4 denote the heights of the assumed layers in the two layer model used to fit the reflectivity data. For clarity of the presentation the interface boundaries inside the film are shown by distinct lines, although they are not real separated layers but just regions of different electron density.

fluctuations are replicated to the surface. Therefore, the film thicknesses of both parts of the film are determined by X-ray reflectivity measurement in addition to the measurement with AFM. X-ray reflectivity measurement delivers only information perpendicular to the sample surface ${ }^{35,48,49} \mathrm{~A}$ model assumption of the in-plane structures and composition of the film is necessary to fit the experimental data, because the average density profile of the film influences the reflectivity data. The reflectivity data and the fitted curves are shown in Figure 5. The fringes present in the data represent the thickness of the whole film and the layer thickness if any present. The measurement on the ultrathin part exhibits two fringes before reaching the background noise at higher values of the scattering vector $q$. In contrast, the measurement on the thin part shows several well pronounced fringes representing the total film thickness. These fringes are smeared-out by the roughness of the interfaces and the background at higher $q$ values. The absence of any Bragg-like peak in the X-ray reflectivity data indicates the lack of a well established internal order. In the ordered state the polymer films would consist of a lamellar stack which is oriented parallel to the substrate surface. ${ }^{50}$ In general, such kind of order is installed by annealing processes. In the present investigation, we did not anneal our samples and thus no lamellar structures are expected to form. Moreover, due to the selected size of the nanoparticles as compared to the bulk lamellar spacing of the diblock copolymer, the addition of nanoparticles will prevent the installation of the common lamellar stack. The nanoparticles simply do not fit into the polystyrene part of the lamellae, and a very high entropy penalty prevents the installation of the wellknown ordered structure. Thus, the composite films are highly disordered. As a result, no microphase separation structures are observed in X-ray reflectivity and GISAXS measurement. Such disorder is necessary for establishing the roughness correlation, because the ordered lamellar structure parallel to the substrate would resist the roughness transfer. Higher energy is needed to bend lamellar structures than disordered structures to replicate the roughness spectrum of the substrate to the polymer film surface. ${ }^{52}$ Due to preferential surface enrichment of one component of the diblock copolymer the polystyrene phase stays at the substrate surface and polyisoprene at the air interface, which lead absence of lamellae perpendicular to the substrate for large film thicknesses.

To obtain the density profile, the reflected intensity is calculated as a function of the scattering vector

$$
q=\frac{4 \pi \sin \theta}{\lambda}
$$

using the Parratt fitting software, ${ }^{36}$ where $\theta$ is the incident angle in $\theta-2 \theta$ geometry and $\lambda$ is the wavelength of $\mathrm{X}$-rays.

Because we do not see any sign of a Bragg-like peak and due to the presence of nanoparticles it was assumed for the modeling that there was no more than one real (but heterogeneous) layer of composite materials present in the thin and ultrathin part of the film. However, since the material is a composite of a polymer and nanoparticles, it is obvious that the local density of the material can differ with the depth and with the position (ultrathin and thin part of the film). Because of gravity acting on the solution of polymer and nanoparticles in toluene, deposited on the solid support before starting the spin-coating process, and during the whole spin-coating process as well as the preferential enrichment of polystyrene chains attached to the nanoparticles it is assumed that the particles migrate toward the substrate. As a result, our model of the composite film is taking difference in density with respect to height from the substrate into consideration to fit the X-ray reflectivity data. The schematic representation of the used model for the ultrathin and thin part of the film is shown in Figure 6. The corresponding parameters of the best fit in this simple two layer model are listed in Table 1. The volume composition and the mean position of the particles are extracted from the average electron density of individual layers obtained from the fitting.

(a) Ultrathin Part. The ultrathin part of the film has a total average thickness of $7.9 \mathrm{~nm}$. It consists of two layers (denoted $h 1$ and h2) of different electron density, i.e., two different compositions. From the average electron density of each layer ( $h 1$ and $h 2$ ) we calculated the composition of both layers (see Figure $6 \mathrm{a}$ ). The bottom layer, $h 2=4.2 \mathrm{~nm}$, consists of polymer and nanoparticles. The volume fraction of nanoparticles in this bottom layer is 0.365 and that of $\mathrm{P}(\mathrm{S}-b-\mathrm{I})$ is 0.635 calculated 
from the average electron density. The chemically attached PS chains are automatically included in the volume fraction of polymer. The height of this upper layer is $h 1=3.7 \mathrm{~nm}$. From its fitted electron density the volume fraction of the nanoparticles is still 0.365 but no more polymer is present in this layer. Thus, the remaining upper parts of the nanoparticles, which are not embedded in the copolymer, form this upper layer. In the upper layer the chains grafted to the nanoparticles are collapsed because air is a bad solvent for PS (not shown in Figure 6a) and their minor contribution is neglected in the simple model. Because the nanoparticles are bigger than the polymer layer in the ultrathin part, the nanoparticles are only partially embedded in the copolymer. Of course, although two different average electron densities, i.e., two different layers are drawn in Figure $6 \mathrm{a}$, in reality there is only one layer containing nanoparticles partially covered by polymer and partially by air describing the ultrathin film part. A higher number of layers in the model could fit the data better than the presented case but it will not change the overall distribution of the composite film in the ultrathin film part as shown in Figure 6a. Therefore, we restricted to the presented two layer model to fit the data and to extract the average depth profile of the film. The model does not include directly the big clusters present in the film because they do not contribute to the X-ray reflectivity signal.

(b) Thin Part. The thin part of the film has also been fitted by assuming two layers of different electron densities (see Figure 6b). The total thickness of the film is $68 \mathrm{~nm}$. The bottom layer $h 4$ has a thickness of $17 \mathrm{~nm}$ and top layer $h 3$ has a thickness of $51 \mathrm{~nm}$. Both layers differ in the electron density and thus resemble different amounts of nanoparticles. The volume fractions of nanoparticles are 0.357 and 0.027 in bottom $h 4$ and upper $h 3$ layer respectively. The shallow holes in the copolymer film surface are just taken into account by an increase in roughness. Figure $6 \mathrm{~b}$ shows a sketch to picture the distribution of the nanoparticles as a function of depth. Of course, the splitting of the film in two layers is artificial and a three layers model might slightly improve the fit quality. However, these layers are not really separated layers but one layer of different densities because of different concentration of nanoparticles. As expected, the nanoparticles are enriched at the substrate surface and the thickness of this enrichment layer is slightly larger than the diameter of the nanoparticles (hydrodynamic diameter in a good solvent $\sim 11 \mathrm{~nm}$ ) to account the presence of particles in a second row (as indicated in Figure 6b). The increased $\mathrm{Si}$ substrate roughness accounts in the simplified model for the fact that the particles have a spherical shape. Since this simplified model can describe our film composition and the X-ray reflectivity data, we did not use any more complicated model.

By comparing the amount of nanoparticles in the ultrathin and the thin film it is obvious that particles have enriched in the ultrathin region. Of course mass conservation is valid but difficult to relate with the concentration used for the spincoating, because of the unknown losses of nanoparticles during the spin-coating process itself.

3. Long-Range Correlations. In reflectivity measurements, only averaged information on the density profile are detected. To probe interface correlations a non-specular intensity needs to be recorded. We have selected grazing incidence small-angle $\mathrm{X}$-ray scattering (GISAXS) in combination with a moderate microfocused X-ray beam and scanning of the sample with respect to the beam. The basic working principle of the GISAXS setup is shown in Figure 2 and the details of the scanning scheme are described in the experimental section. The measure- ment is performed at an incident angle of $\alpha_{i}=0.72^{\circ}$, which is above the critical angle of the polymer and nanoparticles used in the system. As a result, the beam penetrates the whole sample depth and gives rise to the typical scattering features like Yoneda peak, specular peak and modulation due to resonant diffuse scattering (in the case of interface correlation). ${ }^{22,23}$ The scattered intensities of the individual position of measurement of the X-ray beam from the sample are recorded on a $2 \mathrm{~d}$ detector. The measured scattering patterns are shown for the different positions as a composite image in Figure 7 (left). To focus on the modulation of the intensities along the vertical direction (at $q_{y}$ $=0$ ), starting near the Yoneda region, in Figure 7(left) a zoomin part of the $2 \mathrm{~d}$ intensity is selected. As we can see, that the ultrathin part (position -6 to -1 ) of the film shows no modulation in intensities in the selected region. Position 0 corresponding to the rim shows the first intensity modulation along the vertical direction. With further scanning toward the thin part of the film this modulation continues to appear until the end of the scan (position 1 to 13). If the film is uncorrelated all interfaces scatter independently and the diffuse intensities of all individual interfaces superpose. In the case of a fully or partially correlated film roughness, a partial coherence of the diffusely scattered intensity causes resonant diffuse scattering. The partial phase coherence of the waves diffusely scattered from different interfaces concentrates the intensity in narrow sheets. As a result, modulations of intensity are generated. Such modulations in the intensity are observed in the thin part of the film. As a consequence, the investigated $700 \mu \mathrm{m}$ long thin film part of the sample shows long range interface correlation. Assuming simple roughness correlation of the interfaces, the spacing of the fringes in such modulation gives an estimation of the distance between the correlated interfaces $d^{\text {corr } 31}$ by the following one-dimensional Bragg condition: ${ }^{16}$

$$
\Delta q_{z}=\frac{2 \pi}{d^{\mathrm{corr}}}
$$

In addition to the simple case of correlated roughness, interference fringes can also originate from dynamical features of the scattering caused by the refraction of the incident wave. ${ }^{51}$ In this case, the incident wave is reflected from the top surface inside the film. Because the incident and exit waves fulfill the diffraction condition the wave is guided through the film just like as in a typical waveguide. This means coherent reflection of diffusely scattered waves occurs. As a result, Bragg-like resonant lines are observed in the scattering pattern. The most common and pronounced dynamical features is the Yoneda peak. The waveguide behavior can easily be distinguished from the roughness correlation because they differ in their wavelength. The distance in the waveguide case $d^{\text {dyn }}$ can be calculated by the $2 \mathrm{~d}$ Bragg equation as following:

$$
\Delta q_{z}=\frac{\pi}{d^{\mathrm{dyn}}}
$$

To determine long ranged correlations, such as correlated roughness or a waveguide behavior of the film, it is necessary to analyze the $2 \mathrm{~d}$ scattering pattern by performing cuts along the vertical direction, i.e., the $q_{z}$ component of the scattering vector.

Therefore, a vertical slice at $q_{y}=0$ integrating over a small $\Delta q_{y}$ from each of the $2 \mathrm{~d}$ scattering pattern is made and plotted in Figure 7(right) to determine the correlated thickness ( $d^{\text {corr }}$ or $\left.d^{\text {dyn }}\right)$. These vertical cuts are frequently called detector scans. At the specular position the intensity drops due to shielding by 

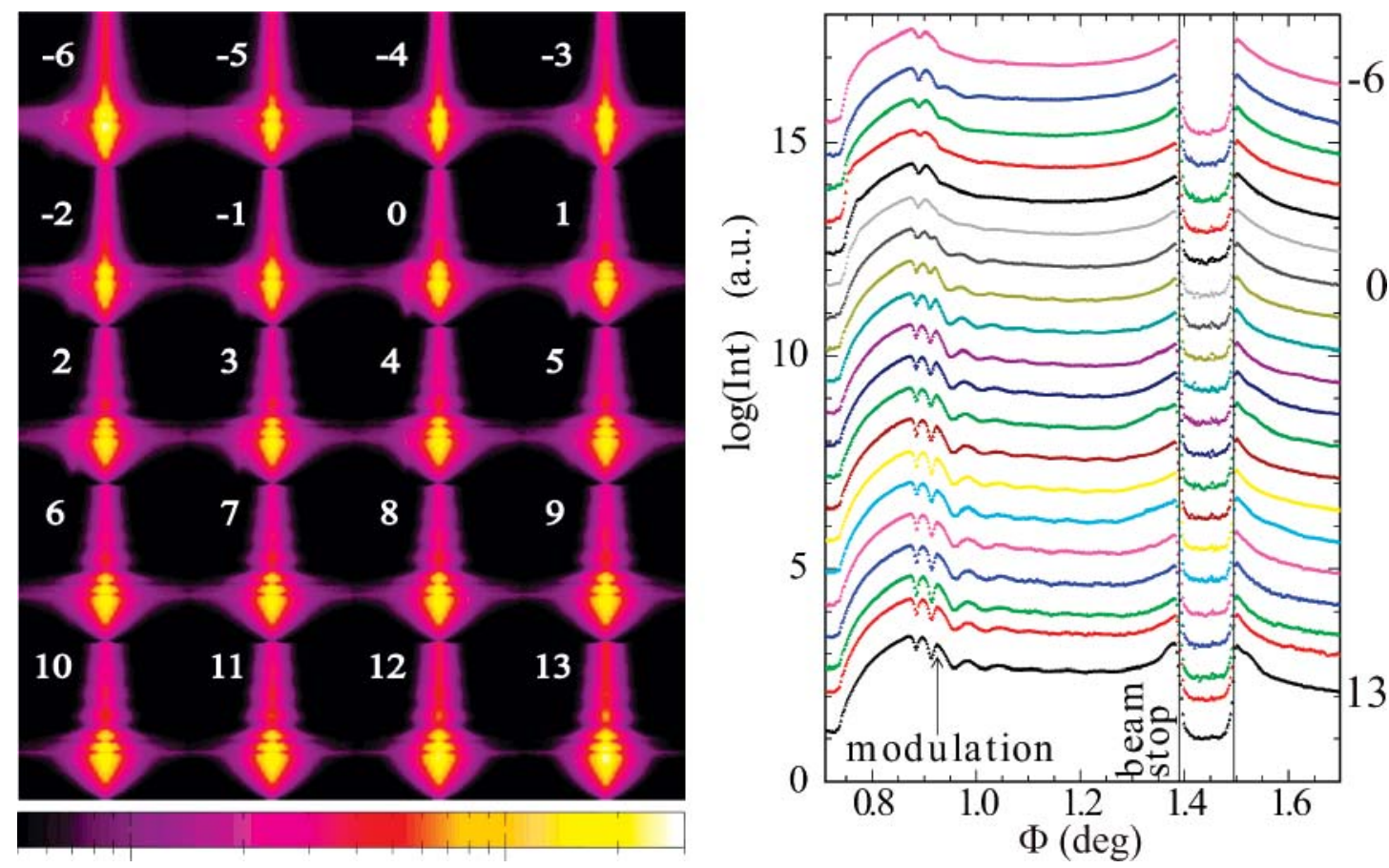

Figure 7. (Left) Composite image comprising the $2 \mathrm{~d}$ scattering patterns from 20 positions of measurement starting from ultrathin part ( -6 to -1 ) through the thickness gradient rim (0) to the thin part of the film (1 to 13). To focus on the modulation a zoom-in part of the whole scattering pattern on the detector is selected. The color scale is selected as in logarithmic for showing better contrast of the present features. The numbers present in each image are the measurement position on the film. Modulation fringes are present in $\mathrm{q}_{z}$ direction in all scattering patterns of rim and thin part of the film (position 0 to 13). Ultrathin part has no modulation fringes. (Right) Detector cuts of all scattering patterns at $q_{y}=0$. The scattering intensities are plotted against the detector angle, $\Phi=\alpha_{i}+\alpha_{f}$ where $\alpha_{i}$ is the incident angle and $\alpha_{f}$ is the exit angle. The curves are shifted along vertical direction neglecting the intensity change for clear understanding. The intensity drop at the specular peak position is due to the presence of beam stop shown by two vertical solid lines. The numbers on the right vertical axis correspond to the position of measurements. This means the ultrathin part is on top of the plot and the thin part is at the bottom separating by the rim at 0 .

the second beam stop. The cuts show that there is a well-defined modulation in the intensity between the Yoneda and the specular peak (shielded) representing a roughness replication in thin part and rim of the film. Consequently, the roughness of the substrate is reproduced by the composite film surface. Thus, although nanoparticles are embedded in the diblock copolymer film, a long-ranged interface correlation is present. Both interfaces, the substrate and composite surface, are correlated starting from large lateral lengths down to a critical cutoff length even in presence of nanoparticles. The ultrathin part of the film is independent to roughness correlation and shows no modulation fringes in detector cuts (position -6 to -1 ). The clear explanation for this behavior is that the ultrathin part is not a continuous film but dewetted polymeric structures containing nanoparticles and their clusters of sizes larger than that of host. As a result, on top of the surface the roughness spectrum has waves of amplitude larger than that present in the roughness spectrum of the substrate. This phenomenon prevents strongly the roughness replication to the surface as it was already observed in the case of simple homopolymer films without added nanoparticles. ${ }^{22}$

The correlated thickness, $d^{\mathrm{corr}}$ or $d^{\mathrm{dyn}}$, of the film is the length of propagation of correlations in the vertical direction. This length can be given by the thickness of the whole film or by a few layers in multilayered films. Moreover, roughness correlation or waveguide like behavior can both occur. To distinguish between both, we have used eqs 2 and 3 to calculate the long range correlation length for each measurement position. The ultrathin part has no modulation fringes giving rise to no correlated thickness. $d^{\text {corr }}$ and $d^{\text {dyn }}$ values were measured for thin part and rim of the film from the average distance between two successive peak positions of the modulation fringes present in detector cuts. The values are plotted against corresponding

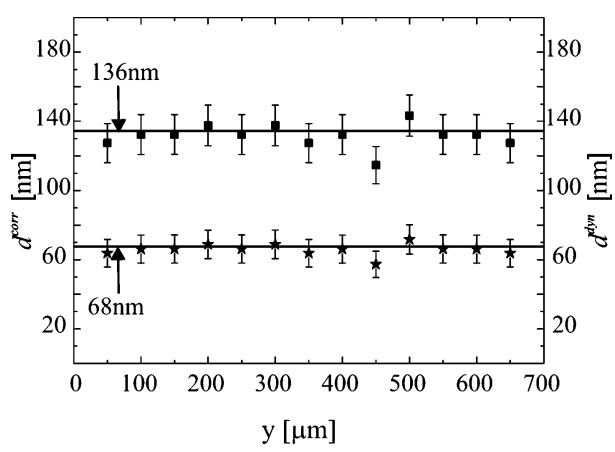

Figure 8. Extracted correlated thickness of the thin part of the film plotted vs the distance from the rim position (defined 0 ). $d^{\text {corr }}$ is calculated by eq 2 and $d^{\text {dyn }}$ by (3). Total film thickness in the shown region of the film is $68 \mathrm{~nm}$ which is equal to the $d^{\text {dyn }}$ values.

distance from the rim position (0) in Figure 8. Within the experimental error, the data are almost constant at all position of measurement in thin part of the film. The average $d^{\text {corr }}$ is $\sim 136 \mathrm{~nm}$ and $d^{\mathrm{dyn}}$ is $\sim 68 \mathrm{~nm}$. The $d^{\text {corr }}$ and $d^{\mathrm{dyn}}$ vales are calculated according to the Bragg equation (eq 2) and the modified Bragg equation for waveguide (eq 3), respectively. Since the thin part of the film has a total thickness of $68 \mathrm{~nm}$, it is obvious that the correlated thickness cannot be $136 \mathrm{~nm}$, and we can exclude the presence of simple roughness correlation. Instead, $d^{\text {dyn }}$ values are in very good agreement with the total film thickness. Therefore, we can conclude that the whole thin part of the film has a roughness correlation in a waveguide manner or in other words the whole thin part is acting as a waveguide. This means the nanocomposite thin film containing maghemite nanoparticles can guide the incident wave through the film constrained by the substrate and the top surface toward the detector. So far our knowledge, this is the first observation 


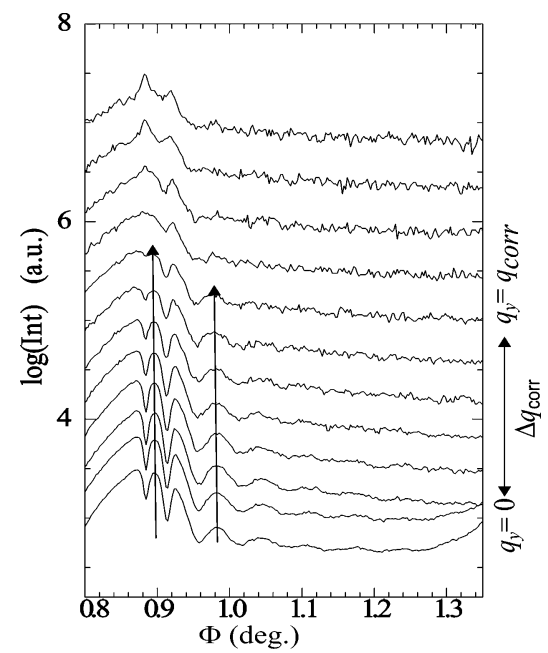

Figure 9. Example of $R_{\mathrm{c}}$ determination. The vertical cuts from the $2 \mathrm{~d}$ intensity at different $q_{y}$ positions are plotted against the angle, $\Phi=\alpha_{i}$ $+\alpha_{\mathrm{f}}$ where $\alpha_{\mathrm{i}}$ is the incident angle and $\alpha_{\mathrm{f}}$ is exit angle. For a better presentation the curves are shifted along vertical axis without maintaining the scale. At larger $q_{y}$ the modulation decreases. No modulation is seen at $q_{y}=q_{\text {corr }}$. The value of $\Delta q_{\text {corr }}$ gives directly the in-plane cutoff length scales $R_{\mathrm{c}}$ via eq 4 . The propagation of modulation is shown by two arrows.

of such kind of behavior in the case of polymer nanocomposite film. The nanoparticles, which are enriched close to the substrate and which are supposed to change the roughness spectrum, did not disturb this type of long range correlation. The possible reason might be the presence of PS chains, which did not change the roughness spectrum but acted like an ultrathin layer on the substrate. In a previous study, it was observed that the same copolymer film without any nanoparticles, having different film thicknesses, showed correlated roughness but not the waveguide behavior. ${ }^{34}$ Therefore, this kind of waveguide effect is due to the presence of high-density nanoparticles in the present system.

4. Lateral Cutoff Length, $\boldsymbol{R}_{\mathbf{c}}$. Because of the conformation of the polymer chains, not all lateral structure sizes of the roughness spectrum of the substrate can be replicated by a polymer film. The quality of roughness replication for different polymeric film can be compared by the critical lateral cutoff length $R_{\mathrm{c}} . R_{\mathrm{c}}$ is defined as the smallest lateral structure length that is replicated on top of the surface of the film, i.e., the substrate morphology is replicated by the thin polymer film for in-plane length scales $R>R_{\mathrm{c}}$. The in-plane cutoff length scale, $R_{\mathrm{c}}$ is determined from the decay of the intensity modulations as a function of $q_{y}$. Thus, vertical cuts parallel to detector cut are selected from the $2 \mathrm{~d}$ intensity at different $q_{y}$ components (see Figure 9). As is visible in Figure 9 the amplitude of the modulations decreases with increasing $q_{y}$ (from bottom to top). The critical cutoff length is calculated by eq 4 as follows: ${ }^{52}$

$$
R_{\mathrm{c}}=\frac{2 \pi}{\Delta q_{\mathrm{corr}}}
$$

where the $\Delta q_{\text {corr }}$ is the lateral (along $q_{y}$ ) propagation length of the modulation arisen due to roughness replication or waveguide effect in reciprocal space in GISAXS data.

Thus, $R_{\mathrm{c}}$ values at all positions of measurement except the ultrathin part are determined and plotted against their relative position from the rim shown in Figure 10. At the rim position, the $R_{\mathrm{c}}$ is very high $(345 \mathrm{~nm})$. This $R_{\mathrm{c}}$ decreases immediately as we move away from the rim in the thin film region. Then it becomes almost constant $(98 \mathrm{~nm})$. The rim height is $700 \mathrm{~nm}$ which is the clear explanation for its higher $R_{\mathrm{c}}$ since it increases

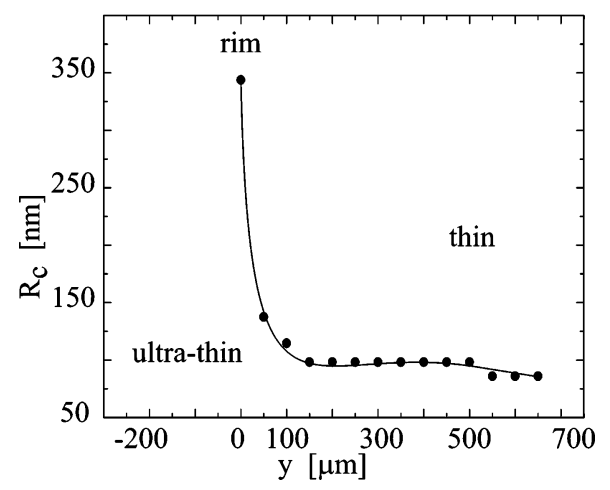

Figure 10. Critical cutoff length $R_{\mathrm{c}}$ as a function of the relative distance $\mathrm{y}$ from the rim position. The solid line is the guide line to the eye. The ultrathin part has no roughness correlation and therefore no $R_{\mathrm{c}}$ values are detectable.

with film thickness. ${ }^{23}$ Although the thin part has a constant thickness the $R_{\mathrm{c}}$ values are not identical for all positions of the measurement. This might be because of a variation of the nanoparticle concentration inside the film near the rim. Since the film has been produced by spin-coating only and no further annealing steps are involved the nanoparticle concentration gradient produced during spin-coating by the strong centrifugal force acting on the nanoparticles is frozen-in. During spincoating a very high centrifugal force field parallel to the substrate is created and the nanoparticles are forced to pass through the covered area (ultrathin part). But, because of thickness constraints all particles cannot pass at the same time. They form clusters nearby the rim. These clusters inhibit the transformation of the small waves from the substrate to the surface. As a result, only larger waves are replicated, i.e., high $R_{\mathrm{c}}$ values are detected.

\section{Conclusion}

We report on the controlled preparation of films with two different film thicknesses separated by one high rim-shaped wall with one single preparation step. The films are based on maghemite nanoparticles embedded in a diblock copolymer. We selected the thickness regime of a thin and an ultrathin (inhomogeneous) film which might be most interesting to applications because it includes the possibility to have a homogeneous film (in thin film part) together with an inhomogeneous one (in ultrathin film part). In the ultrathin part, the nanoparticles are freely accessible whereas in the thin film part they are buried inside the polymer film. In the thin film part the nanoparticles enriched close to the substrate surface and thus the concentration of the nanoparticles is not constant through the copolymer film. Moreover, the copolymer is not ordered into a typical microphase separated structure, which is a necessary prerequisite for roughness correlation. The thin part of the film and the thickness gradient rim showed a long ranged correlation between the substrate and the composite film surface. The lateral critical cutoff length $R_{\mathrm{c}}$ for this part is $\sim 98 \mathrm{~nm}$. The interface correlation follows a waveguide behavior. The whole thin part of the nanocomposite film can act as waveguide due to the presence of nanoparticles. Thus, in the thin part of the film a locally constant thickness is established instead of only a mean constant film thickness. On lateral lengths larger than $98 \mathrm{~nm}$ the mean and the local thicknesses are constant. Only for lateral lengths smaller than $98 \mathrm{~nm}$, the mean and the local thickness deviate (as it is commonly the case in thin films which exhibit no long ranged correlation). With respect to the application of thin nanocomposite films this is a key to the possibility of a reduced amount of necessary material. In contrast, in the ultrathin part no roughness replication is present. 
Acknowledgment. We thank the Bavarian State Ministry of Sciences, Research, and Arts for funding this research work through the International Graduate School "Materials Science of Complex Interfaces" (CompInt).

\section{References and Notes}

(1) Lisari, J. J. Plastic Coatings for Electronics; McGraw-Hill: New York 1970.

(2) Cowie, J. M. G. Polymers: Chemistry and Physics of Modern Materials; Chapman and Hall: New York, 1991.

(3) Feng, Y.; Karim, A.; Weiss, R. A.; Douglas, J. F.; Han, C. C. Macromolecules 1998, 31, 484.

(4) Shenton, W.; Pum, D.; Sleytr, U. B.; Mann, S. Nature (London) 1997, $389,585$.

(5) Bockstaller, M.: Kolb, R.; Thomas, E. L. Adv. Mater. 2001, 13, 1783.

(6) Alexandre, M.; Dubois, Mater. Sci. Eng. Rev. 2000, 28, 1.

(7) Akora, P.; Zhang, X.; Varughese, B.; Briber, M. R.; Kofinas, P. Polymer 2005, 46, 5194

(8) Schulz, L.; et al. Manuscript in preparation.

(9) Abul Kashem, M. M.; Perlich, J.; Schulz, L.; Roth, S. V.; Petry, W.; Müller-Buschbaum, P. Macromolecules 2007, 40, 5075.

(10) Park, M. J.; Char, K.; Park, J.; Hyeon, T. Langmuir 2006, 22, 4,1376.

(11) Lauter-Pasyuk, V.; Lauter, H. J.; Gordeev, G. P.; Müller- Buschbaum, P.; Toperverg, B. P.; Jernenkov, M.; Petry, W. Langmuir 2003, 19, 7783.

(12) An, L.; Li, W.; Nie, Y.; Xie, B.; Li, Z.; Zhang, J.; Bai, Y. J. Colloid Interface Sci. 2005, 288, 503

(13) Darling, S. B.; Bader, S. D. J. Mater. Chem. 2005, 15, 4189.

(14) Darling, S. B. Surf. Sci. 2007, 601, 2555.

(15) Lauter-Pasyuk, V.; Lauter, H. J.; Gordeev, G. P.; Müller-Buschbaum, P.; Toperverg, B. P.; Petry, W.; Jernenkov, M.; Petrenko, A.; Aksenov, V. Physica B 2004, 350, e939.

(16) Schubert, D. W.; Dunkel, T. Mat. Res. Innovat 2003, 7, 314.

(17) Barnes, K. A.; Douglas, J. F.; Liu, D. W.; Karim, A. Adv. Colloid Interface Sci. 2001, 94, 83.

(18) Barnes, K. A.; Karim, A.; Douglas, J. F.; Nakatani, A. I.; Gruell, H.; Amis, E. J. Macromolecules 2000, 33, 4177.

(19) Krishnan, R. S.; Mackay, M. E.; Hawker, C. J.; Horn, B. V. Langmuir $\mathbf{2 0 0 5}, 21,5770$.

(20) Mackay, M. E.; Hong, Y.; Jeong, M.; Hong, S.; Russell, T. P.; Hawker, C. J.; Vestberg, R.; Douglas, J. F. Langmuir 2002, 18, 1877.

(21) McGarrity, E. S.; Frischknecht, A.; Mackay, M. E. APS March Meeting Bull. 2007.

(22) Müller-Buschbaum, P.; Stamm, M. Macromolecules 1998, 31, 3686.

(23) Müller-Buschbaum, P.; Gutmann, J. S.; Lorenz, C.; Schmitt, T.; Stamm, M. Macromolecules 1998, 31, 9265.

(24) Tolan, M.; Vacca, G.; Wang, J.; Sinha, S. K.; Li, Z.; Rafailovich, M.; Sokolov, J.; Gibaud, A.; Lorenz, H.; Kotthaus, J. P. Physica B 1996, $221,53$.

(25) Stearns, D. J. J. Appl. Phys. 1992, 9, 4286.

(26) Fullerton, E. E.; Pearson, J.; Sowers, J. H.; Bader, S. D.; Wu, X. Z.; Sinha, S. K. Phys. Rev. B 1993, 48, 17432
(27) Gibaud, A.; Cowlam, N.; Vignaud, G.; Richardson, T. Phys. Rev. Lett. 1995, 74, 3205

(28) Nitz, V.; Tolan, M.; Schlomka, J. P.; Seeck, O. H.; Stettner, J.; Press, W.; Stelzle, M.; Sackmann, E. Phys. Rev. B 1996, 54, 5038.

(29) Andelmann, D.; Joanny, J. F.; Robbins, M. O. Europhys. Lett. 1988, 7, 731 .

(30) Robbins, M. O.; Andelmann, D.; Joanny, J.-F. Phys. Rev. A 1991 $43,4344$.

(31) Holý, V.; Baumbach, T. Phys. Rev. B 1994, 49, 10668.

(32) Mol, E. A.; Shindler, J. D.; Shalaginov, A. N.; de Jeu, H. W. Phys. Rev. B 1996, 54, 536.

(33) Mol, E. A.; Wong, G. C. L.; Petit, J. M.; Rieutord, F.; de Jeu, H. W. Phys. Rev. Lett. 1997, 79, 3439.

(34) Müller-Buschbaum, P. Anal. Bioanal. Chem. 2003, 376, 3.

(35) Parratt, L. G. Phys. Rev. 1954, 55, 359.

(36) Braun, C. Parratt32 or The Reflectivity Tool, version 1.5.2; HMI Berlin, 1997-99.

(37) Roth, S. V.; Döhrmann, R.; Dommach, M.; Kuhlmann, M.; Kröger, I.; Gehrke, R.; Walter, H.; Schroer, C.; Lengeler, B.; MüllerBuschbaum, P. Rev. Sci. Instrum. 2006, 77, 085106.

(38) Barnes, K. A.; Douglas, J. F.; Liu, D. W.; Karim, A. Adv. Colloid Interface Sci. 2001, 94, 83.

(39) Barnes, K. A.; Karim, A.; Douglas, J. F.; Nakatani, A. I.; Gruell, H.; Amis, E. J. Macromolecules 2000, 33, 4177.

(40) Krishnan, R. S.; Mackay, M. E.; Hawker, C. J. Polym. Mater. Sci. Eng. 2004, 91.

(41) Mackay, M. E.; Hong, Y.; Jeong, M.; Hong, S.; Russell, T. P.; Hawker, C. J.; Vestberg, R.; Douglas, J. F. Langmuir 2002, 18, 1877.

(42) Briber, R.; Feng, H.; Lee, V.; Kim, H. C.; Miller, R. APS March Meeting Bull. 2004, R1.035.

(43) Holmes, M. A; Mackay, M. E.; Giunta, R. K. J. Nanoparticle Res. 2007, 9, 753.

(44) Krishnan, R. S.; Mackay, M. E.; Duxbury, P. M.; Pastor, A.; Hawker, C. J.; Van Horn, B.; Asokan, S.; Wong, M. S. Nano Lett. 2007, 7 , 484.

(45) Xavier, J. H.; Sharma, S.; Seo, Y. S.; Isseroff, R.; Koga, T.; White, H.; Ulman, A.; Shin, K.; Satija, S. K.; Sokolov, J.; Rafailovich, M. H. Macromolecules 2006, 39, 2972.

(46) Sharma, S.; Rafailovich, M. H.; Peiffer, D.; Sokolov, J. Nano Lett. 2001, 1, 511.

(47) Russ, J. C. Fractal Surface; Plenum: New York; 1971.

(48) Yoneda, Y. Phys. Rev. 1963, 131, 2010.

(49) James, R. W. The Optical principles of the diffraction of X-rays; Oxbow Press: Woodbridge, CT, 1962.

(50) Busch, P.; Posselt, D.; Smilgies, D.-M.; Rauscher, M.; Papadakis, C. M. Macromolecules 2007, 40 (3), 630.

(51) Holý, V.; Pietsch, U.; Baumbach, T. High Resolution X-ray Scattering from Thin Films and Multilayers; Springer-Verlag: New York, 1999

(52) Müller-Buschbaum, P.; Gutmann, J. S.; Lorenz-Haas, C.; Mahltig, B.; Stamm, M.; Petry, W. Macromolecules 2001, 33, 7463. 